\title{
Factors associated with medication non-adherence among patients on hemodialysis: A cross-sectional study
}

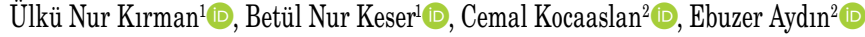 \\ ${ }^{1}$ Faculty of Medicine, Istanbul Medeniyet University, Göztepe Training and Research Hospital, Istanbul, Turkey \\ ${ }^{2}$ Department of Cardiovascular Surgery, Faculty of Medicine, Istanbul Medeniyet University, Göztepe Training and Research Hospital, Istanbul, Turkey
}

Received: July 22, 2020 Accepted: October 02, 2020 Published online: October 21, 2020

\begin{abstract}
Objectives: In this study, we aimed to evaluate the factors associated with medication non-adherence in terms of motivation and knowledge levels among hemodialysis-dependent patients.

Patients and methods: Between March 2018 and April 2018, 134 Caucasian patients (78 males, 56 females; mean age $64.1 \pm 13.0$ years; range, 23 to 86 years) on hemodialysis at two dialysis centers were included in this cross-sectional study. The Modified Morisky Scale was administered to assess the patients' levels of motivation and knowledge about their medications.

Results: The mean time on hemodialysis was $52.3 \pm 50.8$ months and the mean number of prescribed medications was $7.5 \pm 3.5$. The ratio of patients with a low level of motivation and knowledge were $17.2 \%$ and $11.9 \%$, respectively. Hypertension was found to be an independent risk factor for low level of motivation (adjusted odds ratio [aOR]: 5.260 [95\% confidence interval [CI]: 1.122-24.665], $\mathrm{p}=0.035$ ). Being employed was found to be an independent risk factor for low level of knowledge (aOR: 5.000 [95\% CI: 0.404-6.219], p=0.01).

Conclusion: Hypertension and being employed were found to be associated factors of non-adherence to medication therapy. Healthcare professionals should recognize the factors associated with non-adherence and perform effective interventions to prevent adverse outcomes of non-adherence among patients receiving hemodialysis.
\end{abstract}

Keywords: Hemodialysis, knowledge, medication non-adherence, motivation.

Hemodialysis (HD) is a life-saving procedure in the treatment of end-stage renal disease, which requires strict adherence. Adherence in HD therapy has four aspects: fluid restriction, dietary restriction, regular use of prescribed medications, and timely participation to HD sessions. ${ }^{[1]}$ Despite the critical importance of adherence, many patients on HD often fail to follow their recommended treatments. ${ }^{[1,2]}$ According to a recent systematic review study, medication non-adherence is highly frequent in patients receiving $\mathrm{HD}$ with an average rate of $52.5 \% \cdot{ }^{[3]}$

Most of the patients on HD also experience coexisting chronic diseases requiring long-term medication therapy, resulting in multiple medication use. ${ }^{[4]}$ The average number of medication received by the patients is 10 to 12 , while the average number of prescribed medications per day is $19 .{ }^{[3]}$ Moreover, Chiu et al. ${ }^{[5]}$ suggested that patients on HD had the greatest burden of medication among patients with chronic diseases. High burden of medication therapy may lead to non-adherence among patients on HD, and medication non-adherence is related with substantial worsening of medical condition, increased frequency and length of hospitalization, mortality, and increased healthcare costs. ${ }^{[6-8]}$

Motivation and knowledge of patients about their medications have been commonly accepted as good indicators of adherence behavior, while low levels of motivation and knowledge were known to be associated with non-adherence. ${ }^{[9,10]}$ In this present study, we aimed to investigate sociodemographic and clinical risk factors for low levels of motivation and knowledge, which may be associated with nonadherence to medication therapy.

Corresponding author: Ülkü Nur Kırman, MD. İstanbul Medeniyet Üniversitesi Tıp Fakültesi, Göztepe Eğitim ve Araştırma Hastanesi, 34722 Kadıköy, İstanbul, Türkiye.

Tel: +90 216 - 5709416 e-mail: ulkunurkirman@gmail.com

\section{Citation:}

Kırman ÜN, Keser BN, Kocaaslan C, Aydın E. Factors associated with medication non-adherence among patients on hemodialysis: A cross-sectional study. Cardiovasc Surg Int 2020;7(3):129-135. 


\section{PATIENTS AND METHODS}

This cross-sectional study was conducted at Istanbul Medeniyet University, Faculty of Medicine, Department of Cardiovascular Surgery between March 2018 and April 2018. The data of 134 Caucasian patients (78 males, 56 females; mean age $64.1 \pm 13.0$ years; range, 23 to 86 years) receiving $\mathrm{HD}$ at two private dialysis centers were collected. Inclusion criteria were as follows: having a sufficient level of talking, hearing, and cognitive ability to complete the questionnaire and being on HD treatment for more than one month. Exclusion criteria were having advanced dementia or having a caretaker for daily care. To limit participants' fatigue, the study visit was scheduled during the first hour of HD. Necessary permissions were obtained from the Directors of the dialysis centers. A written informed consent was obtained from each patient. The study protocol was approved by the Istanbul Medeniyet University Göztepe Training and Research Hospital Clinical Research Ethics Committee. The study was conducted in accordance with the principles of the Declaration of Helsinki.

\section{Data collection}

The Modified Morisky Scale (MMS) was used, which is the modified version of 4-item Morisky Medication Adherence Scale (MMAS-4 ${ }^{[11]}$ as suggested by the Case Management Society of America ${ }^{[9]}$ It is a well-designed and reliable questionnaire which can separately assess levels of motivation and knowledge, affecting non-adherence behavior to medication therapy. It is a six-item questionnaire and all questions are answered on a "Yes" or "No" scale (Table 1). Additionally, the scale is considerably short and practical to perform in vulnerable patient populations as in this study. The data about the characteristics of the patients were obtained from face-to-face interviews and patients' medical records.

\section{Assessment of medication non-adherence}

On MMS, Items 1, 2, and 6, which measure forgetfulness and carelessness, were considered to be indicators of motivation. Items 3,4 , and 5, which measure discontinuation of medication and understanding of the long-term benefits of the medication, were considered to be indicators of knowledge. ${ }^{[9]}$ If the answer to each question was "Yes", participants would get 0 point; if "No", participants would get 1 point. Participants who received totally $\leq 1$ point from the Items 1,2 , and 6 were assessed as low-motivated and those with $>1$ point were assessed as highly motivated. Participants who received totally $\leq 1$ point from the Items 3,4 , and 5 were assessed to have a low level of knowledge and those with $>1$ point were assessed to have a high level of knowledge. ${ }^{[9]}$

\section{Statistical analysis}

Statistical analysis was performed using the IBM SPSS version 24.0 software (IBM Corp., Armonk, NY, USA). Descriptive data were expressed in mean \pm standard deviation (SD), median (min-max) or number and frequency. The Kolmogorov-Smirnov test was performed to test the normality of the data. Binary logistic regression analysis was conducted to assess the correlations between patient characteristics

\begin{tabular}{|c|c|c|c|}
\hline \multicolumn{4}{|c|}{$\begin{array}{l}\text { Table } 1 \\
\text { Modified Morisky Scale }\end{array}$} \\
\hline & Question & Motivation & Knowledge \\
\hline 1 & Do you ever forget to take your medicine? & Yes (0), No (1) & \\
\hline 2 & Are you careless at times about taking your medicine? & Yes $(0)$, No (1) & \\
\hline 3 & When you feel better do you sometimes stop taking your medicine? & & Yes $(0)$, No $(1)$ \\
\hline 4 & Sometimes if you feel worse when you take your medicine, do you stop taking it? & & Yes $(0)$, No $(1)$ \\
\hline 5 & $\begin{array}{l}\text { Do you know the long-term benefit of taking your medicine as told to you by } \\
\text { your doctor or pharmacist? }\end{array}$ & & Yes $(1)$, No $(0)$ \\
\hline 6 & Sometimes do you forget to refill your prescription medicine on time? & Yes $(0)$, No (1) & \\
\hline & Total score & $\begin{array}{c}- \\
0-1=\text { Low motivation } \\
2-3=\text { High motivation }\end{array}$ & $\begin{array}{c}- \\
0-1=\text { Low motivation } \\
2-3=\text { High motivation }\end{array}$ \\
\hline
\end{tabular}




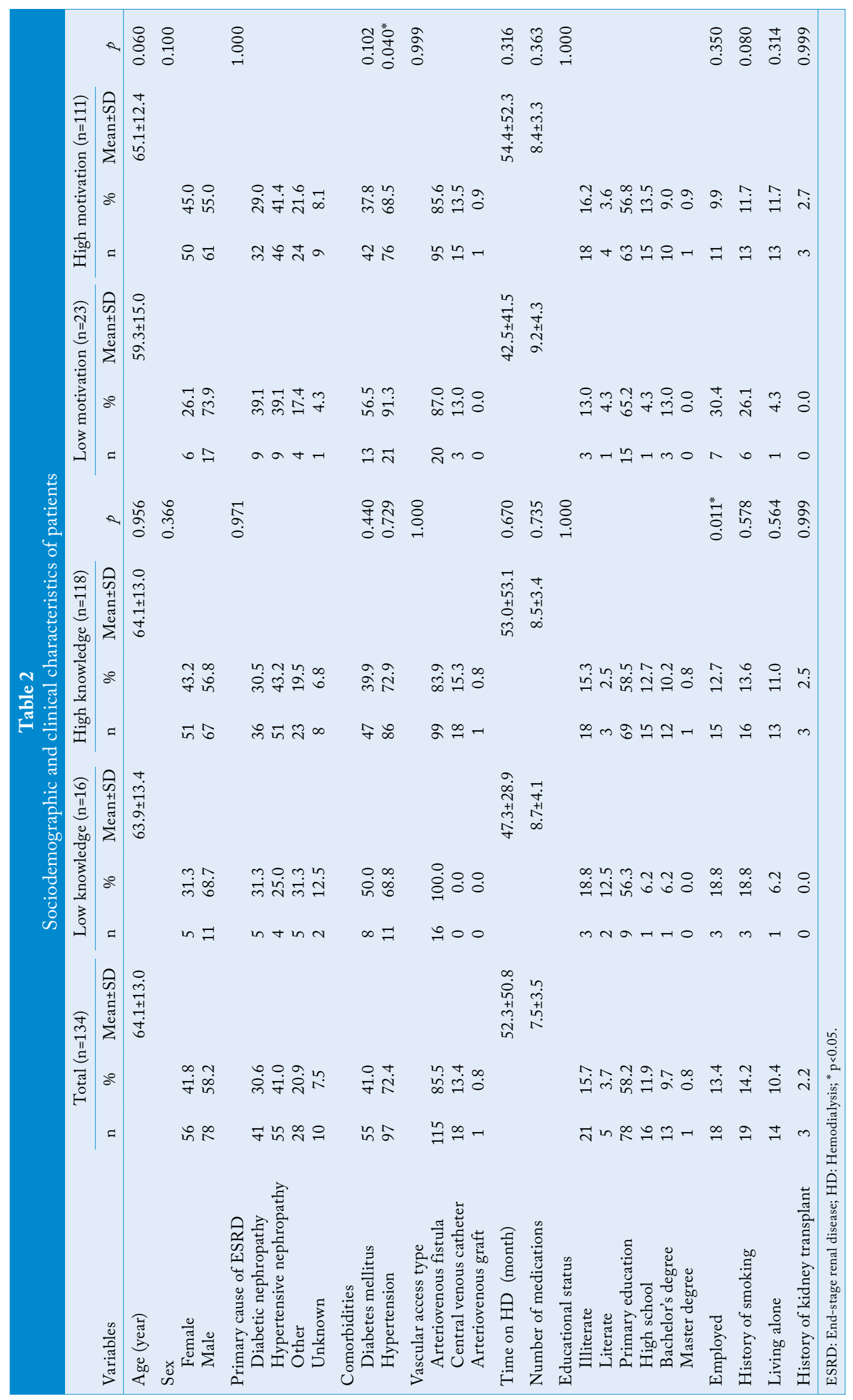




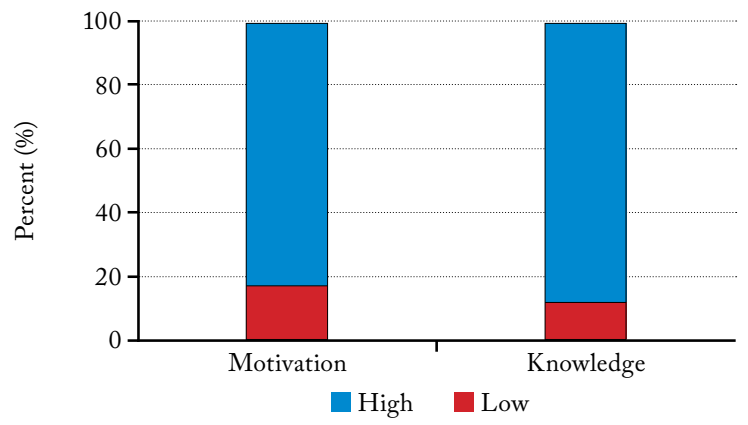

Figure 1. Prevalence of levels of motivation and knowledge of patients.

and levels of motivation and knowledge. On the unadjusted binary logistic regression analyses, the correlations with a significance level below 0.2 were considered confounding factors. To eliminate these factors and to identify the independent risk factors, multiple logistic regression analyses were carried out separately for low level of motivation and low level of knowledge. A two-tailed $p$ value of $<0.05$ was considered statistically significant.

\section{RESULTS}

The mean time on $\mathrm{HD}$ was $52.3 \pm 50.8$ (range, 1 to 296) months and the mean number of prescribed medications was $7.5 \pm 3.5$ (range, 1 to 19 ). Of the participants, $13.4 \%$ were employed and $10.4 \%$ were living alone. Regarding comorbidities, $41.0 \%$ of patients had diabetes mellitus (DM) and $72.4 \%$ had hypertension (HT). All of the patients with HT were on an antihypertensive medication. The patients were on HD via an arteriovenous fistula (85.8\%), central venous catheter (13.4\%), and arteriovenous graft (0.8\%). Baseline sociodemographic and clinical characteristics of the patients are presented in Table 2 .

The ratio of low-motivated patients was $17.2 \%$. The ratio of patients who ever forgot to take their medication was $33.6 \%$. A total of $87.3 \%$ of the patients were careful about the times of taking their medication, while $18.7 \%$ of the patients sometimes forgot to refill their prescribed medications on time. The ratio of patients who had a low level of knowledge was $11.9 \%$. Also, $13.4 \%$ of the patients sometimes stopped taking their medication, if they felt better, whereas $14.9 \%$ of the patients sometimes stopped taking their medication, if they felt worse. A

\begin{tabular}{|lccc|}
\hline \multicolumn{4}{|c|}{ Table 3} \\
Associated factors of medication non-adherence \\
\hline Variable & OR & $95 \%$ CI & $p$ \\
\hline Employment status & 5.000 & $0.404-6.218$ & 0.011 \\
Hypertension & 5.260 & $1.122-24.665$ & 0.035 \\
\hline OR: Adjusted odds ratio; CI: Confidence interval. & \\
\hline
\end{tabular}

total of $52.2 \%$ patients had no knowledge about the long-term benefits of taking their medication. The prevalence of levels of motivation and knowledge of the patients is demonstrated in Figure 1.

In unadjusted logistic regression analyses, the patients with HT indicated a low level of motivation $(\mathrm{p}=0.04)$, and patients who were employed indicated a low level of knowledge $(\mathrm{p}=0.01)$. Although not statistically significant, elderly tended to be highly motivated $(\mathrm{p}=0.06)$. Other variables were not found to be associated with the level of motivation or knowledge.

After adjustment for potential confounders, the analysis revealed that patients with HT had a 5.3-fold risk of low level of motivation. The patients who were employed were under 5-fold risk of low level of knowledge. Associated factors of medication nonadherence are listed in Table 3.

\section{DISCUSSION}

In the present study, we evaluated the factors associated with medication non-adherence in terms of motivation and knowledgelevels among HD-dependent patients. Our study results demonstrated that the ratio of low-motivated patients was $17.2 \%$ and the ratio of patients with a low level of knowledge was $11.9 \%$. Hypertension and being employed were found to be associated factors of medication non-adherence in patients receiving $\mathrm{HD}$.

In the literature review, we found only one study comparable with our ratio of patients with a low level of motivation and knowledge. The ratio of the patients with a low level of motivation (17.2\%) and knowledge (11.9\%) in our study were higher, compared to the study of Ozturk et al. ${ }^{[12]}$ (13.7\% and 8.8\%, respectively).

A large number of studies have shown that HD patients with comorbidities indicate a low level of adherence. ${ }^{[3,13,14]}$ In our study, we investigated HT and DM, which are the most commonly seen comorbidities 
in these patients. Consistent with the results of recent studies, we demonstrated that patients with HT were less motivated, consequently less adherent to medication therapy. Since all of the patients with HT were on antihypertensive medications, it is unclear whether they were less motivated due to HT itself or due to antihypertensive medications. Still, we attribute the non-adherence to medications. A meta-analysis study conducted in Australia investigated non-adherence to antihypertensive drugs in patients on HD and reported that the drugs potentially contributed hypotension after dialysis treatment, and patients may not have any desire to take these medications due to hemodynamic effects they experience. ${ }^{[3,15]}$ Additionally, a comprehensive review study from United States showed that antihypertensive drugs might have unpleasant side effects and provided limited symptomatic relief; therefore, the drugs could adversely affect adherence behavior of patients receiving HD. ${ }^{[8]}$

Although it did not reach statistical significance, our study demonstrated that younger age might be an independent risk factor for a low level of motivation, consequently for non-adherence to medication therapy. There are many studies in the literature reporting that younger age is a predictor for medication non-adherence. ${ }^{[1,14,16]}$ Possible explanations of this finding include that younger patients may have not accepted that they are affected by a chronic disease and may perceive themselves stronger toward the possible complications of non-adherence. Besides, younger patients may have a more prominent feeling of independence, which can lead to disregard of health problems, and non-adherence behavior. ${ }^{[17]}$ Studies have shown that older patients are more aware of possible complications and have more tendency to be adherent. ${ }^{[1,14]}$

In the current study, employed patients had a low level of knowledge, consequently low level of adherence to medication therapy, which is similar to the results of previous studies. ${ }^{[1,6,18]}$ Patients who are working may often fail to follow their prescribed medications due to their business and occupational status. They are more involved in daily living activities and may not be willing to pay attention to the requirements of medication therapy, and tend to be more non-adherent. ${ }^{[1]}$

Studies on the effect of education level on medication non-adherence yield controversial results. Although some studies reported a higher adherence in patients with a higher education level, ${ }^{[4,7]}$ there are studies that have found no association. ${ }^{[1,14]}$ Likewise, we found no statistically significant relationship between education level and medication non-adherence. Of note, patients may not be able to adapt adequately to medications due to some psychosocial reasons, despite their high education level.

A higher number of medication was found to be a significant predictor of medication non-adherence in many studies. ${ }^{[3-5]}$ Due to drug interactions and adverse effects of medications, patients often have difficulty in following their medication therapy. ${ }^{[3,5]}$ Additionally, complexity of medication therapy related to the frequency and dosage schedules is significantly associated with non-adherence. ${ }^{[3]}$ Nonetheless, we found no statistically significant correlation between the number of medication and medication nonadherence which is probably due to having a relatively small sample in the present study.

The results of this study have a number of implications for clinical practice. Healthcare professionals should be aware of factors associated with medication non-adherence in this special patient group, and appropriate adherence improvement plans should be implemented to increase the effectiveness of the treatment.

The Case Management Society of America has suggested adherence guidelines to enhance adherence to medication therapy among patients on HD and explained motivation and knowledge improvement tools in details separately. Long-term benefits of medication therapy and potential consequences of non-adherence should be explained to patients with a low level of knowledge and to their families/personal caregivers via regular educational programs. ${ }^{[9]}$ Patients with a low level of motivation should be managed with motivational interviewing, social support plan, patient reminder systems, and family motivational assessment. ${ }^{[9]}$ Additional adherence improvement methods may reduce complexity of medication therapy, maintenance of relationship between patients and healthcare providers, and early diagnosis of cognitive impairment. ${ }^{[19]}$ Many studies have suggested various adherence improvement recommendations for these patients. However, being aware of factors associated with non-adherence and determining patients' levels of motivation and knowledge should be the initial step in managing non-adherence.

Nonetheless, there are some limitations to this study. First, medication non-adherence was not directly assessed within the frame of this study. 
The scale used in this study demonstrated the risk factors of low level of motivation and knowledge of the patients about their medications, and low level of motivation and knowledge were associated with medication non-adherence among patients. Second, we have a relatively small study group. The other limitation is that we investigated only HT and DM, which are two major comorbidities among patients on HD. Despite the limitations, this study has a significant contribution to the literature by highlighting motivation and knowledge perspectives of medication non-adherence among patients on HD. Other scales evaluating medication non-adherence other than MMS were not originally designed to assess the underlying causes of nonadherence behavior. However, approaching the nonadherence problem in the perspectives of motivation and knowledge is essential to establish appropriate adherence improvement plans that correspond to the patients' specific needs. Still, a limited number of studies has been published considering these perspectives of medication non-adherence. Our study, thus, promotes researchers to focus on patients' motivation and knowledge levels.

In conclusion, HT and being employed were found to be independent risk factors for a low level of motivation and a low level of knowledge, respectively. Based on these results, HT and being employed seem to be associated factors of medication non-adherence in patients receiving HD. However, future studies should be conducted for further understanding of factors associated with medication non-adherence among patients on HD. Approaching the non-adherence problem in the perspectives of motivation and knowledge is essential to design appropriate adherence improvement plans.

\section{Acknowledgements}

We would like to thank Ahmed Turab Akduman, Ilkim Yayla, Merve Ornek, and Muhammet Rasit Tutal for their valuable support with the collection of the data; and Handan Ankarali from the Biostatistics Department for her valuable support in statistical analysis.

\section{Declaration of conflicting interests}

The authors declared no conflicts of interest with respect to the authorship and/or publication of this article.

\section{Funding}

The authors received no financial support for the research and/or authorship of this article.

\section{REFERENCES}

1. Chan YM, Zalilah MS, Hii SZ. Determinants of compliance behaviours among patients undergoing hemodialysis in Malaysia. PLoS One 2012;7:e41362.

2. Kim Y, Evangelista LS, Phillips LR, Pavlish C, Kopple JD. The End-Stage Renal Disease Adherence Questionnaire (ESRD-AQ): testing the psychometric properties in patients receiving in-center hemodialysis. Nephrol Nurs J 2010;37:377-93.

3. Ghimire S, Castelino RL, Lioufas NM, Peterson GM, Zaidi ST. Nonadherence to medication therapy in haemodialysis patients: A systematic review. PLoS One 2015;10:e0144119.

4. Alikari V, Tsironi M, Matziou V, Babatsikou F, Psillakis K, Fradelos E, et al. Adherence to Therapeutic Regimen in Adults Patients Undergoing Hemodialysis: The Role of Demographic and Clinical Characteristics. Int Arch Nurs Health Care 2018;4:096.

5. Chiu YW, Teitelbaum I, Misra M, de Leon EM, Adzize T, Mehrotra R. Pill burden, adherence, hyperphosphatemia, and quality of life in maintenance dialysis patients. Clin J Am Soc Nephrol 2009;4:1089-96.

6. Saran R, Bragg-Gresham JL, Rayner HC, Goodkin DA, Keen ML, Van Dijk PC, et al. Nonadherence in hemodialysis: associations with mortality, hospitalization, and practice patterns in the DOPPS. Kidney Int 2003;64:254-62.

7. Browne T, Merighi JR. Barriers to adult hemodialysis patients' self-management of oral medications. Am J Kidney Dis 2010;56:547-57.

8. Osterberg L, Blaschke T. Adherence to medication. N Engl J Med 2005;353:487-97.

9. Powell SK; Case Management Society of America. The case management adherence guidelines. Lippincotts Case Manag 2005;10:1-2.

10. Sabaté E. Adherence to long-term therapies: evidence for action. Geneva: World Health Organization; 2003.

11. Morisky DE, Green LW, Levine DM. Concurrent and predictive validity of a self-reported measure of medication adherence. Med Care 1986;24:67-74.

12. Özkurt S, Sağlan Y, Gölgeli H, Sağlan R, Balcıoğlu H, Bilge $U$, et al . Assessment of compliance with treatment in hemodialysis patients. Ankara Medical Journal 2017;17:275-83.

13. Rolnick SJ, Pawloski PA, Hedblom BD, Asche SE, Bruzek RJ. Patient characteristics associated with medication adherence. Clin Med Res 2013;11:54-65.

14. Kauric-Klein Z. Predictors of nonadherence with blood pressure regimens in hemodialysis. Patient Prefer Adherence 2013;7:973-80.

15. Sulowicz W, Radziszewski A. Pathogenesis and treatment of dialysis hypotension. Kidney International 2006;70:S36-S39.

16. Naalweh KS, Barakat MA, Sweileh MW, Al-Jabi SW, Sweileh WM, Zyoud SH. Treatment adherence and perception in patients on maintenance hemodialysis: a cross - sectional study from Palestine. BMC Nephrol 2017;18:178. 
17. Mollaoğlu M, Kayataş M. Disability is associated with nonadherence to diet and fluid restrictions in end-stage renal disease patients undergoing maintenance hemodialysis. Int Urol Nephrol 2015;47:1863-70.

18. Smith K, Coston M, Glock K, Elasy TA, Wallston KA, Ikizler TA, et al. Patient perspectives on fluid management in chronic hemodialysis. J Ren Nutr 2010;20:334-41.

19. Schmid H, Hartmann B, Schiffl H. Adherence to prescribed oral medication in adult patients undergoing chronic hemodialysis: a critical review of the literature. Eur J Med Res 2009;14:185-90. 